\title{
Qual o papel das geotecnologias na estruturação de um novo paradigma da Geografia?
}

\section{What is the role of geotechnologies in the structuring of a new geography paradigm?}

\author{
Gabriela Dambrós \\ Doutora em Geografia \\ Professora do Departamento de Geografia da UFPel, Brasil \\ gabbydambros@yahoo.com.br
}

\begin{abstract}
Resumo
A sociedade contemporânea é atingida em todos as dimensões por recentes inovações tecnológicas. O capital e as diversas formas de comunicação imprimem uma nova dinâmica de tempo/espaço que extrapolam fronteiras. $\mathrm{Na}$ mesma velocidade em que essa dinâmica evolui, o espaço geográfico incorpora novas tecnologias, se reorganiza e consolida cada vez mais o meio técnico-científicoinformacional. No contexto da Geografia, a globalização juntamente com a revolução técnicocientífica, possibilita o acesso a uma gama de ferramentas, conhecidas como geotecnologias, que permitem a elaboração, sobretudo de representações cartográficas, mais complexas e precisas. Nessa perspectiva, o presente artigo apresenta reflexões sobre a importância das geotecnologias e a possibilidade de constituição de um novo paradigma geográfico.
\end{abstract}

Palavras-chave: Geotecnologias; Paradigma; Geografia.

\begin{abstract}
Contemporary society is affected in all dimensions by recent technological innovations. Capital and the various forms of communication create a new dynamic of time/space that goes beyond borders. At the same speed as this dynamic evolves, the geographic space incorporates new technologies, reorganises itself and consolidates more and more the technical-scientific-informational environment. In the context of Geography, globalisation together with the technical-scientific revolution allows access to a range of tools, known as geotechnologies, which allow the elaboration, especially of more complex and precise cartographic representations. In this perspective, this article presents reflections on the importance of geotechnologies and the possibility of constituting a new geographical paradigm.
\end{abstract}

Keywords: Geotechnologies; Paradigm; Geography.

\section{INTRODUÇÃO}

Com a globalização, as constantes transformações ocorridas na sociedade vêm oportunizando novas práticas à medida em que a evolução tecnológica, em muitas áreas, perpassa diretamente a construção de novos conhecimentos.

A sociedade contemporânea é atingida, em todos os aspectos, por recentes inovações tecnológicas, o capital e as diversas formas de comunicação imprimem uma nova dinâmica de tempo/espaço que extrapolam fronteiras. Na mesma velocidade em que essa dinâmica evolui, o 
espaço geográfico incorpora novas tecnologias, se reorganiza e consolida cada vez mais o meio técnico-científico-informacional ${ }^{1}$.

No contexto da Geografia, a globalização juntamente com a revolução técnico-científica, possibilita o acesso a uma gama de ferramentas, conhecidas como geotecnologias, que permitem a elaboração, sobretudo de representações cartográficas mais complexas e precisas.

O surgimento das técnicas de sensoriamento remoto e o desenvolvimento da cartografia em ambiente computacional possibilitaram ao geógrafo (e a outros profissionais) trabalhar com um conjunto de informações cada vez maior. Essas tecnologias permitem a aquisição, representação e análise de informações sobre o espaço geográfico sendo que os dados obtidos se tornam importantes para diversas pesquisas.

Nesse sentido, no presente artigo esboça-se algumas questões a partir do entendimento de que as geotecnologias tem potencial para constituir-se como um novo paradigma da Geografia. Busca-se investigar as bases epistemológicas, os avanços proporcionados por estas técnicas na Ciência Geográfica e os principais autores e contribuições. Para tanto, inicialmente faz-se uma abordagem sobre a concepção de paradigma e na sequência discute-se as geotecnologias como técnica e potencial novo paradigma geográfico.

\section{APONTAMENTOS PRELIMINARES SOBRE PARADIGMAS}

Ao longo do período de consolidação do saber geográfico como ciência e sua consequente sistematização, predominaram diferentes correntes teórico-metodológicas. Os desdobramentos e transformações são características normais do processo de construção do conhecimento científico. É neste contexto que emerge a discussão sobre os paradigmas.

Kuhn (1989, p. 13) aponta que paradigmas² são “as realizações científicas universalmente reconhecidas que, durante algum tempo, fornecem problemas e soluções modelares para uma comunidade de praticantes de uma ciência".

Um aspecto importante da teoria de Thomas Kuhn reside na ênfase dada ao caráter revolucionário do progresso científico. Conforme o autor, o progresso se dá mediante saltos e não de forma linear. A forma como Kuhn vê o progresso científico implica na abordagem de alguns conceitos fundamentais como "paradigma", "ciência normal", "anomalia", e "revolução".

A fase que precede a formação da ciência é caracterizada por atividades diversas e por uma desorganização que só mediante a adoção de um paradigma se estrutura. O paradigma constitui-se,

\footnotetext{
${ }^{1}$ Segundo Santos (1997, p. 44) o meio técnico-científico-informacional é um meio geográfico onde o território inclui obrigatoriamente ciência, tecnologia e informação.

${ }^{2}$ Kuhn (1989, p.219) também define paradigma como sendo "[...] aquilo que os membros de uma comunidade partilham e, inversamente, uma comunidade científica consiste em homens que partilham um paradigma”.
} 
assim, em uma estrutura mental assumida que serve para classificar o real antes do estudo ou investigação mais profunda, o que comporta elementos de natureza metodológico-científica, mas também metafísica, e psicológica.

A ciência normal configura-se como o período de atuação dentro de um paradigma que se difundiu por uma comunidade científica. Nessa fase, as pesquisas buscam solucionar problemas que o paradigma assumido detecta. No decorrer das investigações, os pesquisadores podem encontrar problemas que o paradigma não consegue resolver, as chamadas "anomalias" (KUHN, 1989).

A emergência de um novo paradigma se torna necessária quando as anomalias extrapolam o controle e o "modelo" adotado passa a não ser capaz de apresentar respostas satisfatórias. Nesse momento inicia-se a revolução científica, alterando a forma de olhar a realidade, os métodos e instrumentos de pesquisa. Após a adoção de um novo paradigma inicia-se um período de ciência normal até que uma nova crise se instale.

Santos (1978, p.156) entende paradigma como sendo "uma guia para a elaboração de conceitos, teorias e modelos". Quanto às mudanças de paradigma, Santos (1978, p.159) comenta que

\begin{abstract}
Na verdade, basta que tenha mudado a tecnologia para que a estrutura social também mude e, com ambas, a própria teoria. Uma mudança de paradigma corresponde a uma mudança completa na visão do mundo, que o novo paradigma deve representar. Em verdade, não é a nossa visão do mundo que mudou; o que mudou foi o próprio mundo. A história humana é marcada por saltos quantitativos e qualitativos, que significam uma nova combinação de técnicas, uma nova combinação de forças produtivas e, em consequência, um novo quadro para as relações sociais.
\end{abstract}

Dessa forma, pode-se afirmar que um paradigma se assemelha a um modelo, um padrão que uma determinada comunidade científica adota e segue, constituindo-se como um pressuposto filosófico que orienta as pesquisas. Toda ciência precisa de um paradigma, pois é isto que a caracteriza e a torna ciência, juntamente com um objeto de estudo, um método específico e uma filosofia norteadora.

As mudanças de paradigma na ciência sempre estiveram acompanhadas da ideia de superação de modelos, em que se rompe com uma visão antiga e surge uma nova forma de fazer ciência.

A ciência geográfica ao longo de seu desenvolvimento orientou seus estudos, e teorias através de diferentes paradigmas, que originaram as suas Escolas ${ }^{3}$. Nessa perspectiva, pode-se dizer que, cada Escola aceitou uma "verdade geográfica", um paradigma diferente. Embora, ainda existam

\footnotetext{
${ }^{3}$ De acordo com Bezzi; Marafon (2005): Geografia Tradicional ou Clássica (do final do século XIX até 1920/1930), Geografia Quantitativa ou Teorética ou Pragmática ou Nova Geografia (de aproximadamente 1940 ao final da década de 1960), Geografia Crítica ou Radical ou Marxista ou Geografia Nova (a partir do final da década de 1970). Além destas Escolas existem ainda estudos que caracterizam novas tendências atuais e futuras na Geografia.
} 
divergências entre autores sobre o atual paradigma da Geografia, uma das concepções aceitas é a de que a Geografia pode estar constituindo hoje o paradigma Geotecnológico.

\section{GEOTECNOLOGIAS: APENAS TÉCNICA OU NOVO PARADIGMA?}

Para que a discussão sobre geotecnologias tenha sucesso é importante contextualizá-las no tempo, no espaço e na Ciência Geográfica.

No decorrer da evolução do pensamento geográfico vivencia-se uma modernização tecnológica e a busca pelo apreensão da realidade espacial incorpora cada vez mais técnicas. Nesse sentido, Santos (1996, p.25) afirma que "é por demais sabido que a principal forma de relação entre o homem e a natureza, ou melhor, entre o homem e o meio, é dada pela técnica".

As tecnologias de análise espacial emergiram na Geografia Quantitativa avançando devido ao surgimento e difusão dos computadores. Esta Escola Geográfica teve sua gênese relacionada as transformações econômicas, sociais, científicas e tecnológicas do período pós Segunda Guerra Mundial (década de 1950) e baseou seus estudos em métodos e técnicas estatísticas e matemáticas.

Na década de 1960 surgiram os primeiros Sistemas de Informação Geográfica (SIGs) e com estes obteve-se as primeiras visões de automação das tarefas geográficas por meio da computação e a sucessão dos sistemas de softwares e hardware que ganharam ênfase nos estudos e maior aplicabilidade nos anos 1990, sendo capaz de contemplar temas diversos e com monitoramento em tempo real.

No entanto, os SIGs eram muito difíceis de usar, uma vez que não existiam monitores gráficos de alta resolução, os computadores necessários eram caros, e a mão de obra deveria ser altamente especializada. No decorrer dos anos 1970 esse perfil se alterou com o desenvolvimento de novos recursos de hardware, tornando-os mais acessíveis. Nesse mesmo período a expressão Geographic Information System (GIS) tornou-se um conceito através de publicações científicas (CÂMARA; DAVIS, MONTEIRO, 2001).

A partir da década de 1980 a utilização dos SIG passou a crescer de forma mais acelerada. No Brasil o geoprocessamento começou a ser utilizado nesta década, com os primeiros estudos realizados na Universidade Federal do Rio de Janeiro.

Conforme Buzai (2006, p. 582) "los sistemas de información geográfica dejan de ser um simple conjunto de técnicas digitales destinadas a la realización de procedimientos de analisis espacial, para llegar a convertirse em una interfaz de notable carga teórica”.

Na Geografia e em outras áreas, o uso do SIG cresceu nas últimas décadas e passou a figurar como importante ferramenta para aquisição, representação e análise de informações sobre o espaço geográfico. Essas tecnologias permitem tratar dados provenientes de fontes diversas, como por 
exemplo, redes de monitoramento por satélite (imagens, sistema de posicionamento global), levantamentos de campo (topográficos, censitários), mapeamentos sistemáticos, mapeamentos temáticos, com escala de abrangência que vai do local ao global. Os dados obtidos apresentam formatos diferentes e podem expressos na forma de mapas, imagens, relatórios, gráficos, entre outros.

Muitas pesquisas da Geografia estão alicerçadas na utilização de conceitos e métodos sob ambiente computacional, no qual as técnicas de georeferenciamento e cartografia digital tornam-se importantes ferramentas para as pesquisas em diversas áreas do conhecimento geográfico.

Os estudos referentes ao espaço geográfico necessitam de uma vasta gama de conhecimentos e informações que podem ser trabalhadas a partir do uso dos SIGs de forma mais rápida, fácil e precisa por meio de um conjunto de tecnologias implementadas em ambientes computacional responsáveis pelo armazenamento, coleta, recuperação e manipulação de dados.

De acordo Florenzano (2005, p.24): “As geotecnologias referentes ao Sensoriamento Remoto e aos Sistemas de Informações Geográficas estão cada vez mais interligadas. Suas aplicações nos diferentes campos do conhecimento têm aumentado. A princípio, em Geografia essas tecnologias têm uma vasta aplicação".

Pode-se perceber a eficiência destes equipamentos para mapear, controlar, localizar e conhecer áreas de difícil acesso por via terrestre com uma precisão elevada ainda mais quando associadas aos documentos, cartas e mapas gerados de forma analógica.

Dentre as tecnologias de análise espacial destaca-se também o geoprocessamento que de acordo com Rocha (2000, p.210) configura-se como

[...] uma tecnologia transdisciplinar, que através da axiomática da localização e do processamento de dados geográficos, integra várias disciplinas equipamento, programas, processos entidades dados, metodologias e pessoas para coleta, tratamento análise e apresentação de informações associadas a mapas digitais georeferenciados.

As geotecnologias estão cada vez mais, presentes no cotidiano das pessoas e estão sendo adotadas tanto por usuários individuais quanto pelas grandes corporações, subsidiando estudos localizados em pequenas áreas ou espaços abrangentes, até mesmo na escala do globo.

Diante das novas dinâmicas que (re)organizam o espaço geográfico as geotecnologias emergem como importantes recursos tecnológicos frente aos desafios impostos à ciência. Nesse sentido, se faz importante e necessário entender o conceito de geotecnologias, as quais, conforme Rosa (2005, p. 81) são 
[...] o conjunto de tecnologias para coleta, processamento, análise e oferta de informações com referência geográfica. As geotecnologias são compostas por soluções em hardware, software e peopleware que juntos constituem poderosas ferramentas para tomada de decisões. Dentre as geotecnologias podemos destacar: sistemas de informação geográfica, cartografia digital, sensoriamento remoto, sistema de posicionamento global e a topografia.

Essas tecnologias de informação começaram a ser empregadas na Geografia Quantitativa e ao longo do tempo a cartografia digital, sensoriamento remoto, sistema de posicionamento global (GPS), geoprocessamento, entre outras foram ampliando a inserção nas pesquisas geográficas.

Para Dobson (1983 apud Buzai, 2003, p.34) "la geotecnología presenta uma visíon digital del mundo para su tratamiento y análisis mediante los médios computacionales y recién iniciados los ochenta aparece uma primera reflexión sobre su rol em la Geografia como uma revolución tecnológica $[\ldots] "$.

Buzai $(1998,2000,2003,2004)$ defende que as geotecnologias se apresentam como um novo paradigma da Geografia. O autor (1998, p. 190) destaca que "el Paradigma Geotecnológico tiene existencia, pero no como paradigma de la Geografía, sino como paradigma geográfico de alcance interdisciplinario y al servicio total del hombre".

Nesse contexto, pode-se afirmar que a Geografia se estende as demais ciências através da geotecnologia constituindo o que Buzai (1998) denomina como Geografia Global, ou seja, uma Ciência Geográfica difundida por meios informatizados e praticada por profissionais de diferentes áreas que "fazem geografia" sem ser geógrafos.

Ainda de acordo com Buzai (1998, p. 185)

La amplia difusión geotecnológica y su utilización generalizada en actividades de alta valorización contextual han posibilitado la aparición de un sector profesional de usuarios que, independientemente de la disciplina de las cuales provienen, pueden apoyarse en estas tecnologías y "hacer" Geografía; la Geografía que se ha difundido por la inclusión de sus conceptos y métodos en el ambiente computacional para llegar al resto de las ciencias y prácticas sociales mediante procedimientos metodológicos standard. De esta forma se llega al surgimiento de un campo teórico y metodológico de aplicación generalizada; la Geografía Global

A globalização tem sido de fundamental importância para a Geografia na difusão das técnicas ao passo que propicia a troca de informações e construção de novos conhecimentos. Juntamente com o acesso ao computador surgiu a rede mundial de computadores (Internet), que possibilita o conhecimento de um novo e importante espaço, denominado por alguns autores como ciberespaço.

Observa-se, na Figura 1 a trajetória da ciência geográfica, na proposta de Buzai (2004), na qual apresenta os ciclos da evolução do pensamento geográfico. O autor apresenta dois grandes ciclos: 
um período chamado de Ciência Normal subdividido em intervalos de vinte anos ${ }^{4}$ e outro com uma oscilação de um século, que perpassa o século XX chegando ao início do XXI, com destaque para a Geografia Global.

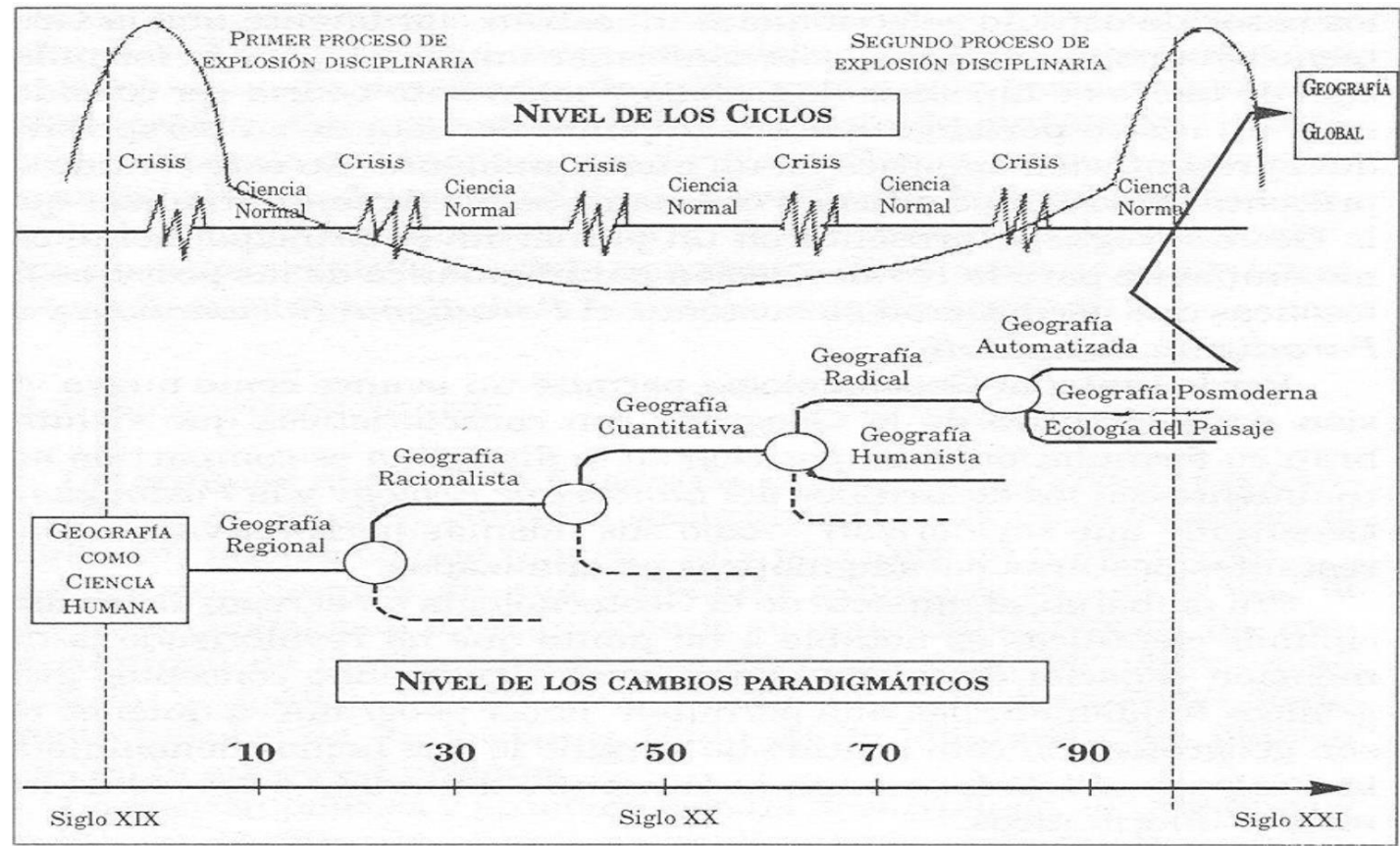

Figura 1 - Trajetória da Ciência Geográfica. Fonte: Buzai, 2004.

Na figura também pode-se visualizar duas grandes explosões ocorridas no pensamento geográfico, as quais Buzai (1998, p.187) caracteriza como

La primera explosión disciplinaria de la Geografía se produce a fines del siglo XIX, la Geografía provee objetos de estudios a diversos campos que surgen del gran proceso de especialización y cien años después se produce la segunda explosión disciplinaria a fines del siglo XX, la Geografía provee conceptos y procedimientos metodológicos standard al resto de las ciencias, el cuerpo teórico que sustenta esta expansión es la Geografía Global y el medio concreto que lo permite es el rol que cumple la Geotecnología.

Com base nos estudos realizados por Buzai pode-se inferir que a Geografia se encontra em um estágio de transição para uma Geografia Global difundida, sobretudo, pela informática, na qual outras ciências se utilizam das geotecnologias para realizar estudos diversos. Seria um período de "popularização" da Ciência Geográfica e, conforme o autor, as geotecnologias consistem no novo paradigma que orienta as pesquisas.

No entanto, é importante questionar: no contexto brasileiro, as geotecnologias são amplamente utilizadas e aceitas como um instrumental técnico importante ou já são compreendidas

\footnotetext{
${ }^{4} \mathrm{O}$ período de ciência normal é rompido por crises e trocas paradigmáticas. O autor enfatiza a Geografia Regional, Geografia Racionalista, Geografia Quantitativa, Geografia Radical, Geografia Humanista, Geografia Automatizada, Geografia Pós-Moderna e Ecologia da Paisagem como concepções alteradas mais ou menos a cada vinte anos.
} 
como um novo paradigma geográfico? Ainda é possível estabelecer mais uma provocação: como as geotecnologias vem sendo utilizadas nos estudos geográficos? Para Breunig et al (2019, p.186) "resistir aos avanços tecnológicos e opor-se aos novos recursos e metodologias têm refletido, ao menos em parte, a atual postura da comunidade geográfica no Brasil". Além disso, os autores apontam que as geotecnologias tem sido empregadas de forma superficial no contexto da Geografia nacional. Responder a todos os questionamentos não é a intenção desse texto, porém acredita-se que as reflexões sobre a utilização das geotecnologias e seus impactos teórico-metodológicos na Geografia são urgentes.

\section{PARA (NÃO) CONCLUIR}

O desenvolvimento da Geografia foi pautado na consolidação e rompimento de distintos paradigmas, o que demonstra o amadurecimento desta ciência. Assim, ao delinear o paradigma Geotecnológico como novo paradigma geográfico, evidencia-se a complexidade da maturação teórica e metodológica da Ciência Geográfica.

As (re)organizações do espaço geográfico ocorrem em virtude dos avanços tecnológicos e dos interesses capitalistas. Esses avanços da tecnologia, para a Geografia, ocorrem mediante o desenvolvimento e utilização de geotecnologias, por meio dos muitos recursos computacionais.

As geotecnologias permitem a aquisição e tratamento de informações espaciais que anteriormente não eram possíveis pelas poucas técnicas disponíveis. Hoje, entretanto, diante dos recursos da informática tais informações estão disponíveis podendo ser acessadas de forma rápida e prática.

A consolidação de um paradigma geotecnológico irá coordenar e dirigir a atividade dos geógrafos nos estudos contemporâneos e futuros? Baseando-se em Buzai pode-se inferir que sim, porém ainda há muitos questionamentos a respeito das geotecnologias e como todo período de crise e emergência de um novo paradigma poucas reflexões aprofundadas têm-se sobre isso. O fato é que as geotecnologias se constituem em recursos que propiciam análises múltiplas e complexas do espaço geográfico.

\section{REFERÊNCIAS}

BEZZI, M. L.; MARAFON, G. J. Historiografia da ciência geográfica. 1. ed. Santa Maria: Ed. da UFSM, 2005. 97p.

BREUNIG, F. M.; HAYAKAWA, E. H.; BACARI, V. M.; TRENTIN, R.; FILHO, W. P.; SILVA, A. Reflexões sobre as geotecnologias no contexto da Geografía no Brasil. Ra'eGa, Curitiba, v. 46, n. 2, p. 185-198, 2019. 
BUZAI, G. D. Impacto de la geotecnología en el desarrollo teórico-metodológico de la ciencia geográfica: hacia un nuevo paradigma en los albores del siglo XXI. 1998. 226 f. Tese (Doutorado em Geografía) - Universidad Nacional de Cuyo, Mendonza, 1998.

La exploración geodigital: implementación, proyecto de investigación e resolución de problemáticas geográficas y medioambientales através de la aplicación de sistemas de información geográfica (sig) con las computadoras personales. 1. ed. Buenos Aires: Lugar, 2000. 179p.

. Mapas Sociales Urbanos. 1. ed. Buenos Aires: Lugar, 2003. 290p.

Geografia Global: el paradigma geotecnológico y el espacio interdisciplinario en la interpretación del mundo del siglo XXI. 1. ed. Buenos Aires: Lugar, 2004. 97p.

Geografia y sistemas de información geográfica. In: HIERNAUX, D.; LINDÒN, A. (Org.). Tratado de Geografia Humana. México: Antrhopos, 2006. 652p.

CÂMARA, G.; DAVIS, C.; MONTEIRO, A. M. V. Introdução à ciência da Geoinformação. 1. ed. São José dos Campos: INPE, 2001. 345p.

FLORENZANO, T. G. Geotecnologias na geografia aplicada: difusão e acesso. Revista do Departamento de Geografia, São Paulo, n. 17, P. 24-29, 2005.

KUHN, T. S. A estrutura das revoluções científicas. 3. ed. São Paulo: Perspectiva, 1989. 324p.

ROCHA, C. H. B. Geoprocessamento: tecnologia transdisciplinar. 1. ed. Juiz de Fora: Ed. do autor, 2000. 220p.

ROSA, R. Geotecnologias na geografia aplicada. Revista do Departamento de Geografia, São Paulo, n. 16, p. 81-90, 2005.

SANTOS, M. Por uma Geografia nova: da crítica da geografia a geografia crítica. 1. ed. São Paulo: HUCITEC, 1978. 288p. $388 \mathrm{p}$.

. A natureza do espaço: técnica e tempo, razão e emoção. 1. ed. São Paulo: HUCITEC, 1996.

.Técnica, espaço, tempo: globalização e meio técnico científico informacional. 4. ed. São Paulo: HUCITEC, 1998. 176p. 